\title{
SPORTS ANTHROPOLOGICAL AND SOMATOTYPICAL COMPARISON BETWEEN YOUNG MALE SHOTPUTTERS AND JAVELIN THROWERS OF DIFFERENT PERFORMANCE CLASSES AND RECREATIONAL ATHLETES
}

\author{
Christoph Raschka, Katrin Vöth, Kim Kuczera \\ Institute of Sports Sciences, Julius Maximilians University Würzburg, Germany
}

\begin{abstract}
This study examines anthropometric and somatotypical differences between German male junior shot putters $(n=19)$ and javelin throwers $(n=19)$.

Recreational athletes $(n=11)$ served as the control group (age range 16 23 years).

Overall, the shot-putters were larger and had longer legs and arms as compared to the junior javelin throwers. For the smaller javelin throwers of the higher performance category shorter legs compared to the height of the hull were observed on average. There was a notably shorter leg of the spear throwers. The powerful javelin throwers had longer arms than the less powerful.

For all anthropometric widths and depths, and the circumferences and the body weight, the junior shout putters achieved higher values than the spear throwers and recreational athletes.

The skinfold thicknesses, the body fat mass and the BMI were highest among the shot putters.

The shot putters had the highest values for endomorphy and pyknomorphia (Knussmann), on the other hand, the lowest values for ectomorphy.

For the shot putters body height, shoulder width, hand width, foot width, chest circumference, minimal forearm circumference, calf circumference, arm span, plastic index and macrosomia were positively related to performance. Some skinfolds (calf, thigh) correlated negatively with the shot put distances.

For the javelin throwers radiale, stylion, dactylion and ectomorphy correlated negatively with the throw widths, but the AKS index, the BMI, the Quetelet index, the Rohrer index, the Livi index, the Pelidisi index, the Broca
\end{abstract}


index, mesomorphy, the metric index and plastic index correlated positively with the performance.

The higher class shot putters of the present study achieved a mean somatotype of $2.9-5.6-1.1$ and the higher class javelin throwers of $2.1-4.9-1.8$. As the shot putters and the javelin throwers of the present investigation were rather young, there is still a significant development potential for the mesomorphic component in comparison to Olympians.

Keywords: sports anthropological investigation, somatotypical investigation, shot putters, junior athletes, javelin throwers, sports anthropometry

\section{INTRODUCTION}

Shot put competitions were first recorded in early 19th century. They were a part of the British Amateur Championships beginning in 1866. The shot put competition for men has been a part of the modern Olympics since their revival in 1896. The first javelin throwing competition at the Olympic Games was held in 1906.

The present study focused on sports anthropometric and somatotypical differences in German junior shot putters and javelin throwers of different performance classes in comparison to recreational athletes.

\section{PARTICIPANTS AND METHODS}

The present study examines anthropometric and somatotypical differences between German male junior shot putters (higher performance level $n=10$, best shot put distance $17.6 \pm 0.9 \mathrm{~m}$, the current shot put distance $17.4 \pm 0.9 \mathrm{~m}$; the lower performance level $n=9$, the best shot put distance $15.1 \pm 1.6 \mathrm{~m}$, the current shot put distance $14.5 \pm 1.7 \mathrm{~m}$; the age range $16-21$ years) and

German male junior javelin throwers (the higher performance level $n=10$, the best throw widths $71.3 \pm 2.7 \mathrm{~m}$, the current throw widths $68.5 \pm 3.1 \mathrm{~m}$; the lower performance level $\mathrm{n}=9$, the best throw widths $57.4 \pm 6.5 \mathrm{~m}$, the current throw widths $55.6 \pm 5.7 \mathrm{~m}$; the age range $16-21$ years).

Recreational athletes (soccer, tennis, hockey) served as the control group (the age range 16-23 years; the lower performance level: the district league $(n=5)$; the higher performance level: the regional league $(n=6))$. Each proband participated voluntarily and the data were used anonymously. Anthropometric data and computed constitutional and somatotypical parameters in this work correspond to international standards (CONRAD 1963, HEATH\&CARTER 1967+1990, KNUSSMANN 1996, MARTIN\&KNUSSMANN 1988, 
RASCHKA 2006, TITTEL\&WUTSCHERK 1972). The analysis of differences was tested by ANOVA, correlations were tested by the Pearson correlation coefficients.

\section{RESULTS}

The distribution of constitutional types after CONRAD and the somatotypes after PARNELL and HEATH \& CARTER are summarized in Figures 1-3.

leptomorph

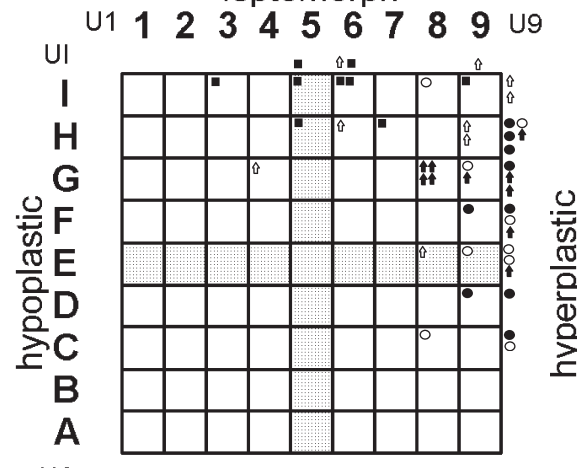

UA

pyknomorph

Figure 1. Shot putters (circles; $n=19)$, javelin throwers (arrows, $n=19$ ), recreational athletes (squares, $\mathrm{n}=11$ ), in the chessboard pattern graphic after CONRAD, higher performance classes (filled, dark symbols), lower performance classes (unfilled blank symbols)

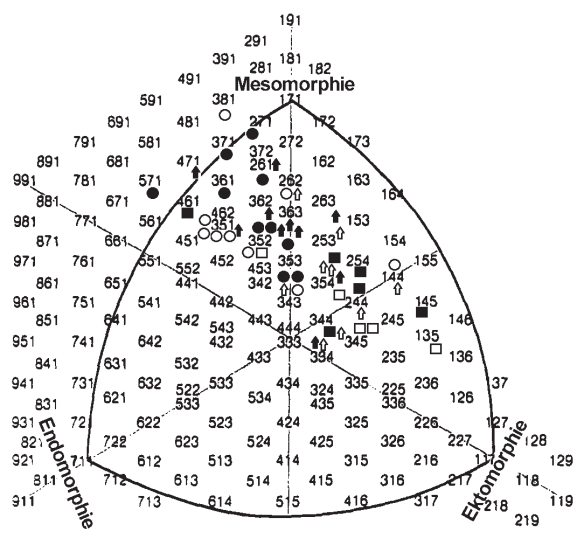

Figure 3. Shot putters (circles; $\mathrm{n}=19$ ), javelin throwers (arrows, $n=19$ ), recreational athletes (squares, $n=11$ ), in the somatochart after HEATH and CARTER, higher performance classes (filled, dark symbols), lower performance classes (unfilled blank symbols)

In the following table all collected anthropometric parameters are listed. 
Table 1. Anthropometric and index parameters of shot putters $(n=19)$, javelin throwers $(n=19)$, recreational athletes $(n=11)$, differentiated as higher performance classes and lower performance classes $\left(p \leq 0.05^{*}, p \leq 0.01^{* *}, p \leq 0.001^{* * *}\right)$

\begin{tabular}{|c|c|c|c|c|c|c|}
\hline \multirow[b]{2}{*}{ Parameter } & \multicolumn{3}{|c|}{ Higher performance level } & \multicolumn{3}{|c|}{ Lower performance level } \\
\hline & $\begin{array}{l}\text { Shot putter } \\
\qquad(n=10)\end{array}$ & $\begin{array}{l}\text { Javelin } \\
\text { thrower } \\
(n=10)\end{array}$ & $\begin{array}{c}\text { Recreational } \\
\text { athletes } \\
(n=6)\end{array}$ & $\begin{array}{l}\text { Shot putter } \\
\qquad(n=9)\end{array}$ & $\begin{array}{l}\text { Javelin } \\
\text { thrower } \\
(n=9)\end{array}$ & $\begin{array}{c}\text { Recreational } \\
\text { athletes } \\
(n=5)\end{array}$ \\
\hline Height (cm) & $189.8 \pm 7.4^{* *}$ & $183.3 \pm 5.7^{* *}$ & $180.0 \pm 6.7^{* *}$ & $188.7 \pm 7.3^{* *}$ & $187.8 \pm 5.7^{* *}$ & $181.4 \pm 4.7^{* *}$ \\
\hline Gnathion $(\mathrm{cm})$ & $164.8 \pm 7.1^{* *}$ & $158.8 \pm 5.3^{* *}$ & $155.9 \pm 6.6^{* *}$ & $164.4 \pm 6.5^{* *}$ & $163.3 \pm 7.2^{* *}$ & $157.0 \pm 4.5^{* *}$ \\
\hline Suprasternale(cm) & $154.6 \pm 6.3^{* *}$ & $148.4 \pm 5.0^{* *}$ & $146.3 \pm 5.9^{* *}$ & $153.9 \pm 6.3^{* *}$ & $152.3 \pm 5.2^{* *}$ & $147.5 \pm 4.3^{* *}$ \\
\hline Acromiale $(\mathrm{cm})$ & $156.1 \pm 6.8^{* *}$ & $149.4 \pm 5.6^{* *}$ & $147.7 \pm 5.5^{* *}$ & $156.1 \pm 5.8^{* *}$ & $154.3 \pm 5.3^{* *}$ & $148.6 \pm 4.1^{* *}$ \\
\hline Radiale $(\mathrm{cm})$ & $119.4 \pm 6.1^{* *}$ & $113.9 \pm 5.0^{* *}$ & $114.1 \pm 4.0^{* *}$ & $120.7 \pm 4.6^{* *}$ & $119.2 \pm 4.1^{* *}$ & $114.4 \pm 3.7^{* *}$ \\
\hline Stylion (cm) & $91.8 \pm 4.7^{*}$ & $87.2 \pm 3.9^{*}$ & $89.1 \pm 2.3^{*}$ & $92.9 \pm 3.7^{*}$ & $92.2 \pm 4.5^{*}$ & $88.8 \pm 2.2^{*}$ \\
\hline Dactylion (cm) & $71.2 \pm 4.6$ & $67.2 \pm 3.8$ & $69.6 \pm 2.3$ & $72.0 \pm 2.5$ & $71.2 \pm 3.7$ & $69.5 \pm 2.4$ \\
\hline Iliocristale (cm) & $115.1 \pm 6.1^{* *}$ & $109.6 \pm 4.8^{* *}$ & $107.9 \pm 5.0^{* *}$ & $115.4 \pm 5.2^{* *}$ & $114.1 \pm 3.9^{* *}$ & $109.2 \pm 2.9^{* *}$ \\
\hline Iliospinale $(\mathrm{cm})$ & $108.3 \pm 5.3^{*}$ & $104.0 \pm 4.7^{*}$ & $102.2 \pm 4.8^{*}$ & $107.7 \pm 4.6^{*}$ & $108.2 \pm 4.4^{*}$ & $103.7 \pm 2.1^{*}$ \\
\hline Trochanterion(cm) & $92.5 \pm 6.1$ & $88.3 \pm 5.3$ & $90.1 \pm 2.1$ & $92.1 \pm 5.7$ & $93.1 \pm 4.6$ & $89.0 \pm 2.9$ \\
\hline Tibiale $(\mathrm{cm})$ & $53.0 \pm 2.4^{*}$ & $51.4 \pm 2.6^{*}$ & $50.3 \pm 3.0^{*}$ & $53.5 \pm 2.8^{*}$ & $54.1 \pm 2.8^{*}$ & $52.2 \pm 3.2^{*}$ \\
\hline Sphyrion $(\mathrm{cm})$ & $9.2 \pm 0.7$ & $9.0 \pm 0.7$ & $8.9 \pm 0.9$ & $8.6 \pm 0.9$ & $9.4 \pm 0.8$ & $9.4 \pm 0.4$ \\
\hline Sitting height(cm) & $97.8 \pm 4.1^{* *}$ & $95.6 \pm 3.4^{* *}$ & $92.6 \pm 3.7^{* *}$ & $96.6 \pm 4.0^{* *}$ & $96.0 \pm 3.1^{* *}$ & $92.9 \pm 3.6^{* *}$ \\
\hline Zygomatic breadth $(\mathrm{cm})$ & $13.6 \pm 0.7$ & $13.3 \pm 1.0$ & $13.3 \pm 0.4$ & $13.8 \pm 0.5$ & $13.4 \pm 0.5$ & $13.1 \pm 0.4$ \\
\hline Morph.facial height(cm) & $12.9 \pm 0.7$ & $12.4 \pm 0.6$ & $12.3 \pm 0.5$ & $12.6 \pm 0.6$ & $12.4 \pm 0.8$ & $12.5 \pm 0.6$ \\
\hline Arm span (cm) & $197.2 \pm 7.7^{* * *}$ & $191.8 \pm 7.0^{* * *}$ & $182.4 \pm 7.9^{* * *}$ & $194.4 \pm 7.4^{* * *}$ & $191.7 \pm 5.1^{* * *}$ & $183.6 \pm 4.9^{* * *}$ \\
\hline Shoulder width (cm) & $46.3 \pm 3.1^{* *}$ & $45.2 \pm 3.0^{* * *}$ & $41.8 \pm 2.7^{* *}$ & $44.1 \pm 2.1^{* *}$ & $42.8 \pm 4.1^{* *}$ & $41.0 \pm 1.8^{* *}$ \\
\hline Chest width $(\mathrm{cm})$ & $33.0 \pm 2.0 * * *$ & $30.0 \pm 1.6^{* * *}$ & $27.8 \pm 2.5^{* * *}$ & $31.4 \pm 1.8^{* * *}$ & $28.4 \pm 2.0^{* * *}$ & $27.6 \pm 1.3^{* * *}$ \\
\hline Chest depth $(\mathrm{cm})$ & $22.3 \pm 2.7^{* * *}$ & $20.5 \pm 1.1^{* * *}$ & $19.7 \pm 1.9^{* * *}$ & $22.6 \pm 3.3^{* * *}$ & $20.0 \pm 1.4^{* * *}$ & $18.3 \pm 1.8^{* * *}$ \\
\hline Pelvis width $(\mathrm{cm})$ & $32.1 \pm 2.6^{* * *}$ & $28.3 \pm 1.2^{* * *}$ & $27.8 \pm 2.0^{* * *}$ & $30.5 \pm 2.6^{* * *}$ & $27.5 \pm 1.3^{* * *}$ & $27.4 \pm 2.0^{* * *}$ \\
\hline $\begin{array}{l}\text { Epiphysis width } \\
\text { Humerus cm }\end{array}$ & $7.1 \pm 0.5^{*}$ & $6.7 \pm 0.5^{*}$ & $6.5 \pm 0.6^{*}$ & $6.9 \pm 0.3^{*}$ & $6.8 \pm 0.5^{*}$ & $6.3 \pm 0.4^{*}$ \\
\hline Radioulnar breadth(cm) & $5.7 \pm 0.3^{* * *}$ & $5.4 \pm 0.3^{* * *}$ & $5.1 \pm 0.2^{* * *}$ & $5.5 \pm 0.3^{* * *}$ & $5.3 \pm 0.3^{* * *}$ & $5.0 \pm 0.1^{* * *}$ \\
\hline Hand breadth $(\mathrm{cm})$ & $8.2 \pm 0.3^{* * *}$ & $7.7 \pm 0.4^{* * *}$ & $7.2 \pm 0.5^{* * *}$ & $8.0 \pm 0.3^{* * *}$ & $7.7 \pm 0.3^{* * *}$ & $7.0 \pm 0.7^{* * *}$ \\
\hline Epiphysis width femur & $10.6 \pm 0.5^{* * *}$ & $9.9 \pm 0.3^{* * *}$ & $9.9 \pm 0.6^{* * *}$ & $10.4 \pm 0.4^{* * *}$ & $9.8 \pm 0.3^{* * *}$ & $9.7 \pm 0.5^{* * *}$ \\
\hline Ancle breadth $(\mathrm{cm})$ & $8.0 \pm 0.1^{* *}$ & $7.7 \pm 0.6^{* *}$ & $7.3 \pm 0.5^{* *}$ & $7.7 \pm 0.4^{* *}$ & $7.6 \pm 0.4^{* *}$ & $7.3 \pm 0.1^{* *}$ \\
\hline Neck circumference(cm) & $43.1 \pm 2.0^{* * *}$ & $41.6 \pm 1.8^{* * *}$ & $37.7 \pm 2.2^{* * *}$ & $40.6 \pm 1.0^{* * *}$ & $39.8 \pm 1.6^{* * *}$ & $37.3 \pm 1.5^{* * *}$ \\
\hline $\begin{array}{l}\text { Chest circumference, } \\
\text { respiratory centre }(\mathrm{cm})\end{array}$ & $112.7 \pm 8.2^{* * *}$ & $103.8 \pm 4.4^{* * *}$ & $95.7 \pm 8.6^{* * *}$ & $105.9 \pm 7.4^{* * *}$ & $97.8 \pm 5.8^{* * *}$ & $91.9 \pm 6.3^{* * *}$ \\
\hline $\begin{array}{l}\text { Chest circumference, } \\
\text { in inspiration }(\mathrm{cm})\end{array}$ & $115.0 \pm 7.9^{* * *}$ & $106.5 \pm 4.7^{* * *}$ & $99.0 \pm 9.0^{* * *}$ & $108.9 \pm 7.0^{* * *}$ & $100.9 \pm 5.9^{* * *}$ & $95.7 \pm 5.6^{* * *}$ \\
\hline $\begin{array}{l}\text { Chest circumference, } \\
\text { in exspiration }(\mathrm{cm})\end{array}$ & $111.8 \pm 7.8^{* * *}$ & $102.1 \pm 4.2^{* * *}$ & $95.3 \pm 8.6^{* * *}$ & $105.4 \pm 7.4^{* * *}$ & $96.7 \pm 5.9 * * *$ & $90.7 \pm 5.3^{* * *}$ \\
\hline Waist circumference cm & $94.3 \pm 8.7^{* * *}$ & $82.9 \pm 2.8^{* * *}$ & $79.1 \pm 5.6^{* * *}$ & $89.1 \pm 7.4^{* * *}$ & $79.5 \pm 4.1^{* * *}$ & $76.8 \pm 5.1^{* * *}$ \\
\hline Pelvis circumference & $99.8 \pm 9.5^{* * *}$ & $86.2 \pm 3.2^{* * *}$ & $85.6 \pm 8.2^{* * *}$ & $94.3 \pm 7.3^{* * *}$ & $84.2 \pm 3.3^{* * *}$ & $83.6 \pm 7.3^{* * *}$ \\
\hline
\end{tabular}




\begin{tabular}{|c|c|c|c|c|c|c|}
\hline \multirow[b]{2}{*}{ Parameter } & \multicolumn{3}{|c|}{ Higher performance level } & \multicolumn{3}{|c|}{ Lower performance level } \\
\hline & $\begin{array}{l}\text { Shot putter } \\
\qquad(n=10)\end{array}$ & $\begin{array}{l}\text { Javelin } \\
\text { thrower } \\
(n=10)\end{array}$ & $\begin{array}{l}\text { Recreational } \\
\text { athletes } \\
(n=6)\end{array}$ & $\begin{array}{l}\text { Shot putter } \\
\qquad(n=9)\end{array}$ & $\begin{array}{l}\text { Javelin } \\
\text { thrower } \\
(n=9)\end{array}$ & $\begin{array}{l}\text { Recreational } \\
\text { athletes } \\
(n=5)\end{array}$ \\
\hline $\begin{array}{l}\text { Upper arm circumf. in } \\
\text { extension }(\mathrm{cm}) \text { right side }\end{array}$ & $36.4 \pm 2.2^{* * *}$ & $33.5 \pm 1.9^{* * *}$ & $30.2 \pm 2.1^{* * *}$ & $35.1 \pm 3.2^{* * *}$ & $31.0 \pm 2.0^{* * *}$ & $28.7 \pm 2.6^{* * *}$ \\
\hline $\begin{array}{l}\text { Upper arm circumf. in } \\
\text { flexion }(\mathrm{cm}) \text { right side }\end{array}$ & $38.1 \pm 2.3^{* * *}$ & $35.6 \pm 2.1^{* * *}$ & $32.3 \pm 1.6^{* * *}$ & $37.2 \pm 3.0^{* * *}$ & $33.3 \pm 2.3^{* * *}$ & $30.6 \pm 2.5^{* * *}$ \\
\hline $\begin{array}{l}\text { Upper arm circumf. in } \\
\text { extension }(\mathrm{cm}) \text { left side }\end{array}$ & $35.9 \pm 1.9^{* * *}$ & $33.0 \pm 1.7^{* * *}$ & $29.8 \pm 2.3^{* * *}$ & $35.2 \pm 2.7^{* * *}$ & $30.4 \pm 2.3^{* * *}$ & $28.0 \pm 2.5^{* * *}$ \\
\hline $\begin{array}{l}\text { Upper arm circumf. in } \\
\text { flexion }(\mathrm{cm}) \text { left side }\end{array}$ & $37.3 \pm 2.0^{* * *}$ & $35.2 \pm 2.1^{* * *}$ & $31.5 \pm 1.9^{* * *}$ & $36.9 \pm 3.0^{* * *}$ & $32.5 \pm 2.9^{* * *}$ & $29.8 \pm 2.5^{* * *}$ \\
\hline $\begin{array}{l}\text { Forearm circumference } \\
\text { maximum }(\mathrm{cm}) \text { right side }\end{array}$ & $31.4 \pm 1.1^{* * *}$ & $29.8 \pm 1.5^{* * *}$ & $27.0 \pm 1.5^{* * *}$ & $30.7 \pm 1.6^{* * *}$ & $28.3 \pm 1.9^{* * *}$ & $26.4 \pm 1.7^{* * *}$ \\
\hline $\begin{array}{l}\text { Forearm circumference } \\
\text { minimum }(\mathrm{cm}) \text { right side }\end{array}$ & $19.2 \pm 0.4^{* * *}$ & $17.8 \pm 0.8^{* * *}$ & $16.4 \pm 1.3^{* * *}$ & $18.7 \pm 0.9^{* * *}$ & $17.7 \pm 1.0^{* * *}$ & $16.4 \pm 0.6^{* * *}$ \\
\hline $\begin{array}{l}\text { Forearm circumference } \\
\text { maximum }(\mathrm{cm}) \text { left side }\end{array}$ & $30.3 \pm 1.1^{* * *}$ & $29.1 \pm 1.3^{* * *}$ & $26.2 \pm 1.7^{* * *}$ & $30.1 \pm 1.4^{* * *}$ & $27.1 \pm 1.7^{* * *}$ & $25.9 \pm 1.4^{* * *}$ \\
\hline $\begin{array}{l}\text { Forearm circumference } \\
\text { minimum }(\mathrm{cm}) \text { left side }\end{array}$ & $18.9 \pm 0.4^{* * *}$ & $17.8 \pm 0.7^{* * *}$ & $16.1 \pm 1.2^{* * *}$ & $18.5 \pm 0.8^{* * *}$ & $17.2 \pm 0.8^{* * *}$ & $16.1 \pm 0.8^{* * *}$ \\
\hline $\begin{array}{l}\text { Hand circumference }(\mathrm{cm}) \\
\text { dominant side }\end{array}$ & $22.7 \pm 0.7^{* * *}$ & $21.9 \pm 1.0^{* * *}$ & $19.8 \pm 1.3^{* * *}$ & $22.4 \pm 1.0^{* * * *}$ & $21.5 \pm 1.0^{* * *}$ & $20.0 \pm 0.9^{* * *}$ \\
\hline $\begin{array}{l}\text { Thigh circumference } \\
(\mathrm{cm}) \text { right side }\end{array}$ & $66.3 \pm 3.6^{* * *}$ & $61.1 \pm 3.4^{* * *}$ & $55.1 \pm 3.8^{* * *}$ & $64.0 \pm 4.0^{* * *}$ & $57.9 \pm 3.6^{* * *}$ & $52.6 \pm 2.6^{* * *}$ \\
\hline $\begin{array}{l}\text { Thigh circumference } \\
\text { (cm) left side }\end{array}$ & $65.0 \pm 3.1^{* * *}$ & $60.4 \pm 2.9^{* * *}$ & $54.4 \pm 4.0^{* * *}$ & $63.3 \pm 3.7^{* * *}$ & $57.3 \pm 4.0^{* * *}$ & $52.3 \pm 4.0^{* * *}$ \\
\hline $\begin{array}{l}\text { Calf circumference }(\mathrm{cm}) \\
\text { right side }\end{array}$ & $43.0 \pm 2.2^{* * *}$ & $39.5 \pm 2.2^{* * *}$ & $36.6 \pm 3.0^{* * *}$ & $40.2 \pm 2.3^{* * *}$ & $38.2 \pm 2.4^{* * *}$ & $37.2 \pm 1.4^{* * * *}$ \\
\hline $\begin{array}{l}\text { Calf circumference }(\mathrm{cm}) \\
\text { left side }\end{array}$ & $43.0 \pm 2.4^{* * *}$ & $39.9 \pm 1.9^{* * *}$ & $37.0 \pm 3.2^{* * *}$ & $40.9 \pm 2.3^{* * *}$ & $38.5 \pm 2.3^{* * *}$ & $37.8 \pm 1.7^{* * *}$ \\
\hline $\begin{array}{l}\text { Lower leg circumference } \\
(\mathrm{cm}) \text { minimum right side }\end{array}$ & $25.4 \pm 0.8^{* * *}$ & $23.6 \pm 1.2^{* * *}$ & $22.6 \pm 1.5^{* * *}$ & $24.3 \pm 1.4^{* * *}$ & $23.9 \pm 1.7^{* * *}$ & $23.3 \pm 0.8^{* * *}$ \\
\hline $\begin{array}{l}\text { Lower leg circumference } \\
(\mathrm{cm}) \text { minimum left side }\end{array}$ & $25.8 \pm 0.9^{* * *}$ & $24.0 \pm 1.1^{* * *}$ & $22.5 \pm 2.1^{* * *}$ & $24.7 \pm 1.5^{* * *}$ & $23.9 \pm 1.8^{* * *}$ & $23.5 \pm 0.9^{* * *}$ \\
\hline Anthr. foot length (cm) & $26.9 \pm 1.0^{*}$ & $26.8 \pm 1.1^{*}$ & $25.8 \pm 1.1^{*}$ & $27.4 \pm 1.3^{*}$ & $27.0 \pm 1.2^{*}$ & $24.9 \pm 2.4^{*}$ \\
\hline Techn. foot length $(\mathrm{cm})$ & $25.4 \pm 1.2$ & $25.2 \pm 1.0$ & $24.5 \pm 0.8$ & $25.4 \pm 1.3$ & $25.1 \pm 2.3$ & $24.0 \pm 1.1$ \\
\hline Foot width (cm) & $10.7 \pm 0.6^{* * *}$ & $10.0 \pm 0.4^{* * *}$ & $9.7 \pm 0.5^{* * *}$ & $10.1 \pm 0.6^{* * *}$ & $9.9 \pm 0.5^{* * *}$ & $9.6 \pm 0.4^{* * *}$ \\
\hline $\begin{array}{l}\text { Foot circumference }(\mathrm{cm}) \\
\text { right side }\end{array}$ & $27.4 \pm 1.3^{* * *}$ & $25.6 \pm 0.7^{* * *}$ & $24.6 \pm 1.2^{* * *}$ & $26.4 \pm 1.0^{* * *}$ & $25.8 \pm 1.1^{* * *}$ & $24.9 \pm 0.8^{* * *}$ \\
\hline $\begin{array}{l}\text { Foot circumference }(\mathrm{cm}) \\
\text { left side }\end{array}$ & $27.5 \pm 1.4^{* * *}$ & $25.6 \pm 0.8^{* * *}$ & $24.7 \pm 1.2^{* * *}$ & $26.6 \pm 1.0^{* * *}$ & $25.9 \pm 1.3^{* * *}$ & $24.7 \pm 0.7^{* * *}$ \\
\hline Age (years) & $17.0 \pm 1.1^{*}$ & $18.3 \pm 1.6^{*}$ & $19.8 \pm 3.3^{*}$ & $18.0 \pm 1.7^{*}$ & $17.6 \pm 1.5^{*}$ & $18.8 \pm 2.2^{*}$ \\
\hline Weight (kg) & $106.7 \pm 12.8^{* * *}$ & $86.6 \pm 7.0^{* * *}$ & $75.3 \pm 11.1^{* * *}$ & $98.2 \pm 12.0^{* * *}$ & $82.5 \pm 8.3^{* * *}$ & $72.0 \pm 7.0^{* * *}$ \\
\hline Body fat (\%)Caliper & $11.8 \pm 3.4^{* *}$ & $9.5 \pm 1.5^{* *}$ & $9.7 \pm 3.7^{* *}$ & $11.8 \pm 2.2^{* *}$ & $8.9 \pm 1.0^{* *}$ & $9.0 \pm 1.9^{* *}$ \\
\hline Body fat (kg) Caliper & $9.8 \pm 3.6^{* *}$ & $7.4 \pm 1.4^{* *}$ & $7.5 \pm 3.4^{* *}$ & $9.4 \pm 2.1^{* *}$ & $6.7 \pm 1.0^{* *}$ & $6.9 \pm 2.0^{* *}$ \\
\hline BMI $\left(\mathrm{kg} / \mathrm{m}^{2}\right)$ & $29.6 \pm 3.0^{* * *}$ & $25.8 \pm 1.5^{* * *}$ & $23.2 \pm 2.8^{* * *}$ & $27.6 \pm 2.5^{* * *}$ & $23.4 \pm 2.0^{* * *}$ & $21.9 \pm 2.1^{* * *}$ \\
\hline
\end{tabular}




\begin{tabular}{|c|c|c|c|c|c|c|}
\hline \multirow[b]{2}{*}{ Parameter } & \\
\hline & $\begin{array}{l}\text { Shot putter } \\
\qquad(n=10)\end{array}$ & $\begin{array}{l}\text { Javelin } \\
\text { thrower } \\
(n=10)\end{array}$ & $\begin{array}{l}\text { Recreational } \\
\text { athletes } \\
(n=6)\end{array}$ & $\begin{array}{l}\text { Shot putter } \\
\qquad(n=9)\end{array}$ & $\begin{array}{l}\text { Javelin } \\
\text { thrower } \\
(n=9)\end{array}$ & $\begin{array}{l}\text { Recreational } \\
\text { athletes } \\
(n=5)\end{array}$ \\
\hline Quetelet-I. (g/cm) & $5.6 \pm 0.6^{* * *}$ & $4.7 \pm 0.3^{* * *}$ & $4.2 \pm 0.5^{* * *}$ & $5.2 \pm 0.5^{* * *}$ & $4.4 \pm 0.4^{* * *}$ & $4.0 \pm 0.4^{* * *}$ \\
\hline Rohrer-I. $\left(\mathrm{g} / \mathrm{cm}^{3}\right)$ & $1.56 \pm 0.2^{* * *}$ & $1.41 \pm 0.1^{* * *}$ & $1.29 \pm 0.2^{* * *}$ & $1.46 \pm 0.1^{* * *}$ & $1.25 \pm 0.1^{* * *}$ & $1.21 \pm 0.1 * * *$ \\
\hline Livi-I. $\left({ }^{3} \sqrt{\mathrm{g}} / \mathrm{cm}\right)$ & $2.50 \pm 0.1^{* * *}$ & $2.41 \pm 0.1^{* * *}$ & $2.34 \pm 0.1^{* * *}$ & $2.44 \pm 0.1^{* * *}$ & $2.32 \pm 0.1^{* * *}$ & $2.29 \pm 0.1^{* * *}$ \\
\hline $\mathrm{HWR}(\mathrm{cm} / \sqrt[3]{ } \sqrt{\mathrm{kg}})$ & $40.1 \pm 1.5^{* * *}$ & $41.5 \pm 0.9^{* * *}$ & $42.7 \pm 1.5^{* * *}$ & $41.0 \pm 1.4^{* * *}$ & $43.2 \pm 1.3^{* * *}$ & $43.7 \pm 1.5^{* * *}$ \\
\hline 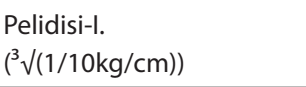 & $104.5 \pm 5.2^{* * *}$ & $99.7 \pm 2.7^{* * *}$ & $98.0 \pm 3.4^{* * *}$ & $102.8 \pm 4.5^{* * *}$ & $97.7 \pm 3.2^{* * *}$ & $96.5 \pm 3.4^{* * *}$ \\
\hline Broca-I. (\%) & $19.0 \pm 12.2^{* * *}$ & $4.0 \pm 6.2^{* * *}$ & $-5.9 \pm 11.1^{* * *}$ & $10.8 \pm 10.3^{* * *}$ & $-6.0 \pm 8.3^{* * *}$ & $-11.4 \pm 8.5^{* * *}$ \\
\hline $\begin{array}{l}\text { Triceps skinfold }(\mathrm{mm}) \\
\text { right side }\end{array}$ & $10.2 \pm 4.3^{* *}$ & $7.0 \pm 3.5^{* *}$ & $6.4 \pm 2.4^{* *}$ & $9.6 \pm 4.3^{* *}$ & $6.1 \pm 1.6^{* *}$ & $5.8 \pm 1.5^{* *}$ \\
\hline $\begin{array}{l}\text { Triceps skinfold (mm) } \\
\text { left side }\end{array}$ & $9.5 \pm 4.2^{* *}$ & $6.7 \pm 3.1^{* *}$ & $6.5 \pm 2.2^{* *}$ & $9.1 \pm 3.1^{* *}$ & $6.1 \pm 1.6^{* *}$ & $6.5 \pm 2.0^{* *}$ \\
\hline $\begin{array}{l}\text { Forearm skinfold (mm) } \\
\text { non dominant side }\end{array}$ & $7.7 \pm 2.7^{* *}$ & $5.3 \pm 1.6^{* *}$ & $5.5 \pm 1.4^{* *}$ & $7.2 \pm 3.5^{* *}$ & $4.7 \pm 0.6^{* *}$ & $4.9 \pm 1.5^{* *}$ \\
\hline $\begin{array}{l}\text { Suprailiac skinfold (mm) } \\
\text { right side }\end{array}$ & $8.1 \pm 3.2^{*}$ & $5.9 \pm 1.6^{*}$ & $7.2 \pm 4.9^{*}$ & $8.3 \pm 2.6^{*}$ & $5.3 \pm 1.5^{*}$ & $6.3 \pm 2.6^{*}$ \\
\hline $\begin{array}{l}\text { Suprailiac skinfold (mm) } \\
\text { left side }\end{array}$ & $8.1 \pm 3.7^{* *}$ & $6.2 \pm 1.5^{* *}$ & $6.4 \pm 4.5^{* *}$ & $8.9 \pm 2.4^{* *}$ & $5.6 \pm 1.2^{* *}$ & $5.5 \pm 2.0^{* *}$ \\
\hline $\begin{array}{l}\text { Subscapular skinfold non } \\
\text { dominant side ( } \mathrm{mm})\end{array}$ & $11.4 \pm 5.2^{* *}$ & $10.3 \pm 1.9 * *$ & $10.1 \pm 3.4^{* *}$ & $12.1 \pm 3.2^{* *}$ & $9.2 \pm 1.7^{* *}$ & $10.0 \pm 3.2^{* *}$ \\
\hline $\begin{array}{l}\text { Thigh skinfold }(\mathrm{mm}) \text { non } \\
\text { dominant side }\end{array}$ & $17.2 \pm 3.2^{* *}$ & $13.9 \pm 4.0^{* *}$ & $11.6 \pm 4.2^{* *}$ & $17.7 \pm 5.3^{* *}$ & $13.0 \pm 3.1^{* *}$ & $13.5 \pm 5.8^{* *}$ \\
\hline $\begin{array}{l}\text { Calf skinfold }(\mathrm{mm}) \text { non } \\
\text { dominant side }\end{array}$ & $13.9 \pm 4.0^{* * *}$ & $10.9 \pm 2.9^{* * *}$ & $9.3 \pm 3.4^{* * *}$ & $16.7 \pm 5.4^{* * *}$ & $10.8 \pm 2.9^{* * *}$ & $11.1 \pm 4.7^{* * *}$ \\
\hline Head height (cm) & $25.0 \pm 1.2$ & $24.6 \pm 0.9$ & $24.1 \pm 1.0$ & $24.2 \pm 1.9$ & $24.5 \pm 2.5$ & $24.4 \pm 0.6$ \\
\hline Neck length (cm) & $10.3 \pm 2.2$ & $10.3 \pm 2.0$ & $9.5 \pm 0.9$ & $10.5 \pm 2.2$ & $11.0 \pm 2.9$ & $9.5 \pm 0.6$ \\
\hline Arm length $(\mathrm{cm})$ & $84.9 \pm 3.3^{* * *}$ & $82.2 \pm 2.5^{* * *}$ & $78.1 \pm 3.7^{* * *}$ & $84.1 \pm 3.5^{* * *}$ & $83.0 \pm 2.6^{* * *}$ & $79.1 \pm 2.5^{* * *}$ \\
\hline $\begin{array}{l}\text { Length of upper arm } \\
\text { and forearm }(\mathrm{cm})\end{array}$ & $64.3 \pm 3.3^{* * *}$ & $62.2 \pm 2.5^{* * *}$ & $58.6 \pm 4.2^{* * *}$ & $63.2 \pm 2.5^{* * *}$ & $62.1 \pm 2.1^{* * *}$ & $59.9 \pm 2.7^{* * *}$ \\
\hline Upper arm length (cm) & $36.7 \pm 1.7^{* *}$ & $35.5 \pm 1.4^{* *}$ & $33.6 \pm 2.0^{* *}$ & $35.4 \pm 1.7^{* *}$ & $35.1 \pm 1.6^{* *}$ & $34.2 \pm 1.5^{* *}$ \\
\hline Forearm length $(\mathrm{cm})$ & $27.6 \pm 2.6^{*}$ & $26.7 \pm 1.8^{*}$ & $25.0 \pm 3.4^{*}$ & $27.8 \pm 1.4^{*}$ & $27.0 \pm 1.8^{*}$ & $25.7 \pm 1.8^{*}$ \\
\hline Hand length (cm) & $20.6 \pm 1.2$ & $20.0 \pm 1.5$ & $19.5 \pm 1.4$ & $20.9 \pm 1.3$ & $21.0 \pm 1.8$ & $19.3 \pm 1.0$ \\
\hline Leg length (cm) & $103.3 \pm 5.3^{*}$ & $99.0 \pm 4.7^{*}$ & $97.2 \pm 4.8^{*}$ & $102.7 \pm 4.6^{*}$ & $103.2 \pm 4.4^{*}$ & $98.7 \pm 2.1^{*}$ \\
\hline $\begin{array}{l}\text { Length of thigh } \\
\text { and calf }(\mathrm{cm})\end{array}$ & $99.1 \pm 5.3^{*}$ & $95.0 \pm 4.2^{*}$ & $93.3 \pm 4.5^{*}$ & $99.1 \pm 4.6^{*}$ & $98.8 \pm 4.4^{*}$ & $94.3 \pm 2.1^{*}$ \\
\hline Thigh length $(\mathrm{cm})$ & $55.3 \pm 3.2^{* *}$ & $52.7 \pm 2.8^{* *}$ & $51.9 \pm 2.2^{* *}$ & $54.2 \pm 2.3^{* *}$ & $54.1 \pm 2.2^{* *}$ & $51.5 \pm 1.8^{* *}$ \\
\hline Calf length (cm) & $43.8 \pm 2.4$ & $42.4 \pm 2.1$ & $41.4 \pm 2.4$ & $44.8 \pm 2.9$ & $44.7 \pm 2.8$ & $42.8 \pm 3.2$ \\
\hline Metrik-Index/CONRAD & $-0.4 \pm 0.7^{* * *}$ & $-0.8 \pm 0.2^{* * *}$ & $-1.1 \pm 0.5^{* * *}$ & $-0.5 \pm 0.6^{* * *}$ & $-1.3 \pm 0.5^{* * *}$ & $-1.4 \pm 0.4^{* * *}$ \\
\hline Plastik-Index/CONRAD & $100.4 \pm 3.7^{* * *}$ & $97.0 \pm 4.4^{* * *}$ & $88.6 \pm 4.8^{* * *}$ & $97.2 \pm 3.6^{* * *}$ & $92.6 \pm 5.5^{* * *}$ & $87.4 \pm 2.5^{* * *}$ \\
\hline $\begin{array}{l}\text { Pyknomorphy/ } \\
\text { Knussmann }\end{array}$ & $1.5 \pm 2.2^{* * *}$ & $0.0 \pm 1.7^{* * *}$ & $-0.3 \pm 1.7^{* * *}$ & $0.8 \pm 2.0^{* * *}$ & $-1.5 \pm 1.4^{* * *}$ & $-2.4 \pm 0.9^{* * *}$ \\
\hline
\end{tabular}




\begin{tabular}{|c|c|c|c|c|c|c|}
\hline \multirow[b]{2}{*}{ Parameter } & \multicolumn{3}{|c|}{ Higher performance level } & \multicolumn{3}{|c|}{ Lower performance level } \\
\hline & $\begin{array}{l}\text { Shot putter } \\
\qquad(n=10)\end{array}$ & $\begin{array}{l}\text { Javelin } \\
\text { thrower } \\
(n=10)\end{array}$ & $\begin{array}{c}\text { Recreational } \\
\text { athletes } \\
(n=6)\end{array}$ & $\begin{array}{l}\text { Shot putter } \\
\qquad(n=9)\end{array}$ & $\begin{array}{l}\text { Javelin } \\
\text { thrower } \\
(n=9)\end{array}$ & $\begin{array}{l}\text { Recreational } \\
\text { athletes } \\
(n=5)\end{array}$ \\
\hline $\begin{array}{l}\text { Makrosomia/ } \\
\text { Knussmann }\end{array}$ & $8.8 \pm 1.6^{* * *}$ & $7.1 \pm 1.8^{* * *}$ & $4.9 \pm 1.2^{* * *}$ & $7.6 \pm 1.9^{* * *}$ & $6.7 \pm 1.8^{* * *}$ & $3.4 \pm 2.5^{* * *}$ \\
\hline Endomorphy/PARNELL & $4.2 \pm 0.6^{* * *}$ & $3.3 \pm 0.5^{* * *}$ & $3.2 \pm 0.8^{* * *}$ & $3.9 \pm 0.7^{* * *}$ & $3.1 \pm 0.4^{* * *}$ & $3.1 \pm 0.7^{* * *}$ \\
\hline Mesomorphy/PARNELL & $4.8 \pm 0.9^{*}$ & $5.2 \pm 1.1^{*}$ & $4.2 \pm 1.1^{*}$ & $4.8 \pm 1.2^{*}$ & $3.9 \pm 0.7^{*}$ & $3.4 \pm 0.7^{*}$ \\
\hline Ektomorphy/PARNELL & $1.6 \pm 0.7^{* * *}$ & $2.1 \pm 0.7^{* * *}$ & $3.4 \pm 1.4^{* * *}$ & $1.8 \pm 0.8^{* * *}$ & $3.3 \pm 0.9 * * *$ & $3.9 \pm 1.1^{* * *}$ \\
\hline $\begin{array}{l}\text { Endomorphy/ } \\
\text { Heath-Carter }\end{array}$ & $2.9 \pm 1.0^{* *}$ & $2.1 \pm 0.6^{* *}$ & $2.1 \pm 0.9^{* *}$ & $2.7 \pm 0.8^{* *}$ & $1.8 \pm 0.3^{* *}$ & $2.0 \pm 0.7^{* *}$ \\
\hline $\begin{array}{l}\text { Mesomorphy/ } \\
\text { Heath-Carter }\end{array}$ & $5.6 \pm 1.1^{* * *}$ & $4.9 \pm 1.2^{* * *}$ & $4.1 \pm 1.1^{* * *}$ & $5.1 \pm 1.1^{* * *}$ & $3.6 \pm 0.7^{* * *}$ & $3.4 \pm 0.9 * * *$ \\
\hline $\begin{array}{l}\text { Ektomorphy/ } \\
\text { Heath-Carter }\end{array}$ & $1.1 \pm 0.8^{* * *}$ & $1.8 \pm 0.6^{* * *}$ & $2.8 \pm 1.0^{* * *}$ & $1.5 \pm 0.9^{* * *}$ & $3.0 \pm 1.0^{* * *}$ & $3.4 \pm 1.1^{* * *}$ \\
\hline AKS-Index & $1.42 \pm 0.10^{* * *}$ & $1.28 \pm 0.10^{* * *}$ & $1.16 \pm 0.10^{* * *}$ & $1.32 \pm 0.10^{* * *}$ & $1.14 \pm 0.10^{* * *}$ & $1.09 \pm 0.10^{* * * *}$ \\
\hline
\end{tabular}

Overall, the shot-putters were larger and had the longer legs and arms as compared to the junior javelin throwers. For the smaller javelin throwers of the higher performance category shorter legs compared to the height of the hull were observed on average. There was a notably shorter leg of the spear throwers. The powerful javelin thrower had longer arms than the less powerful.

For all anthropometric widths and depths, and the circumferences and the body weight the junior shout putters achieved higher values than the spear throwers and recreational athletes.

The skinfold thicknesses, the body fat mass and the BMI were highest among the shot putters.

The shot putters had the highest values for endomorphy and pyknomorphia (Knussmann), on the other hand, the lowest values for ectomorphy.

In the following table the correlations between performance and anthropometric / somatotypical parameters are listed.

For the shot putters body height, shoulder width, hand width, food width, chest circumference, minimal forearm circumference, calf circumference, arm span, plastic index and macrosomia were positively related to performance. Some skinfolds (calf, thigh) correlated negatively with the shot put distances.

For the javelin throwers radiale, stylion, dactylion and ectomorphy correlated negatively with the throw widths, but the AKS index, the BMI, the Quetelet index, the Rohrer index, the Livi index, the Pelidisi index, the Broca index, mesomorphy, metric index and plastic index correlated positively with the performance. 
Table 2. Correlations between anthropometric parameters, best and current shot put distances, best and current javelin throw widths $\left(p \leq 0.05^{*}, p \leq 0.01^{* *}, p \leq 0.001^{* * *}\right)$

\begin{tabular}{|c|c|c|c|c|}
\hline \multirow{2}{*}{$\begin{array}{l}\text { r } \\
\text { Parameter }\end{array}$} & \multicolumn{2}{|c|}{ Shot putter } & \multicolumn{2}{|c|}{ Javelin thrower } \\
\hline & $\begin{array}{c}\text { best } \\
\text { shot put } \\
\text { distance }\end{array}$ & $\begin{array}{l}\text { current } \\
\text { shot put } \\
\text { distance }\end{array}$ & $\begin{array}{l}\text { best } \\
\text { throw } \\
\text { width }\end{array}$ & $\begin{array}{c}\text { current } \\
\text { throw } \\
\text { width }\end{array}$ \\
\hline Age & -0.14 & $-0.38^{*}$ & 0.29 & 0.27 \\
\hline Height $(\mathrm{cm})$ & $0.38^{*}$ & 0.34 & -0.32 & -0.28 \\
\hline Gnathion (cm) & 0.33 & 0.27 & -0.30 & -0.25 \\
\hline Suprasternale $(\mathrm{cm})$ & 0.35 & 0.33 & -0.32 & -0.26 \\
\hline Acromiale $(\mathrm{cm})$ & 0.30 & 0.28 & -0.33 & -0.30 \\
\hline Radiale $(\mathrm{cm})$ & 0.18 & 0.17 & $-0.46^{*}$ & $-0.44^{*}$ \\
\hline Stylion $(\mathrm{cm})$ & 0.19 & 0.20 & $-0.43^{*}$ & $-0.39 *$ \\
\hline Dactylion (cm) & 0.21 & 0.19 & $-0.45^{*}$ & $-0.44^{*}$ \\
\hline Iliocristale (cm) & 0.26 & 0.25 & $-0.39^{*}$ & -0.37 \\
\hline Iliospinale $(\mathrm{cm})$ & 0.29 & 0.32 & -0.33 & -0.28 \\
\hline Trochanterion $(\mathrm{cm})$ & 0.16 & 0.21 & $-0.42^{*}$ & $-0.40^{*}$ \\
\hline Tibiale (cm) & 0.31 & 0.30 & -0.39 & -0.37 \\
\hline Sphyrion (cm) & 0.30 & 0.36 & -0.22 & -0.15 \\
\hline Sitting height $(\mathrm{cm})$ & 0.36 & 0.25 & -0.13 & -0.10 \\
\hline Zygomatic breadth $(\mathrm{cm})$ & -0.07 & -0.16 & 0.09 & 0.14 \\
\hline Morph.facial height(cm) & 0.20 & 0.27 & -0.05 & 0.00 \\
\hline Arm span $(\mathrm{cm})$ & $0.43^{*}$ & $0.40^{*}$ & 0.12 & 0.13 \\
\hline Shoulder width (cm) & $0.48^{*}$ & $0.48^{*}$ & 0.27 & 0.21 \\
\hline Chest width $(\mathrm{cm})$ & $0.40^{*}$ & 0.37 & 0.37 & 0.36 \\
\hline Chest depth $(\mathrm{cm})$ & 0.03 & 0.00 & 0.31 & 0.28 \\
\hline Pelvis width $(\mathrm{cm})$ & $0.41^{*}$ & 0.36 & 0.26 & 0.30 \\
\hline Spinal distance & $0.43^{*}$ & $0.49^{*}$ & 0.21 & 0.25 \\
\hline Epiphysis width Humerus cm & 0.17 & 0.11 & -0.02 & 0.02 \\
\hline Radioulnar breadth $(\mathrm{cm})$ & 0.47 & 0.43 & 0.06 & 0.03 \\
\hline Hand breadth $(\mathrm{cm})$ & $0.45^{*}$ & 0.31 & 0.16 & 0.07 \\
\hline Epiphysis width femur & -0.03 & -0.03 & 0.14 & 0.16 \\
\hline Ancle breadth $(\mathrm{cm})$ & 0.36 & 0.39 & 0.19 & 0.13 \\
\hline Neck circumference $(\mathrm{cm})$ & $0.57^{* *}$ & $0.54^{* *}$ & $0.50^{*}$ & $0.48^{*}$ \\
\hline Chest circumference, respiratory centre $(\mathrm{cm})$ & $0.44^{*}$ & $0.40^{*}$ & $0.49^{*}$ & $0.50^{* *}$ \\
\hline Chest circumference, in inspiration $(\mathrm{cm})$ & $0.43^{*}$ & $0.39^{*}$ & $0.47^{*}$ & $0.46^{*}$ \\
\hline Chest circumference, in exspiration $(\mathrm{cm})$ & $0.44^{*}$ & $0.40^{*}$ & $0.45^{*}$ & $0.46^{*}$ \\
\hline Waist circumference $\mathrm{cm}$ & 0.36 & 0.30 & $0.46^{*}$ & $0.42^{*}$ \\
\hline
\end{tabular}




\begin{tabular}{|c|c|c|c|c|}
\hline \multirow{2}{*}{$\begin{array}{l}\text { r } \\
\text { Parameter }\end{array}$} & \multicolumn{2}{|c|}{ Shot putter } & \multicolumn{2}{|c|}{ Javelin thrower } \\
\hline & $\begin{array}{c}\text { best } \\
\text { shot put } \\
\text { distance }\end{array}$ & $\begin{array}{l}\text { current } \\
\text { shot put } \\
\text { distance }\end{array}$ & $\begin{array}{l}\text { best } \\
\text { throw } \\
\text { width }\end{array}$ & $\begin{array}{l}\text { current } \\
\text { throw } \\
\text { width }\end{array}$ \\
\hline Pelvis circumference & 0.36 & 0.28 & $0.41^{*}$ & $0.40^{*}$ \\
\hline Upper arm circumf. in extension $(\mathrm{cm})$ right side & 0.25 & 0.16 & $0.50^{*}$ & $0.51^{* *}$ \\
\hline Upper arm circumf. in flexion $(\mathrm{cm})$ right side & 0.23 & 0.13 & $0.47^{*}$ & $0.46^{*}$ \\
\hline Upper arm circumf. in extension $(\mathrm{cm})$ left side & 0.22 & 0.12 & $0.47^{*}$ & $0.48^{*}$ \\
\hline Upper arm circumf. in flexion $(\mathrm{cm})$ left side & 0.17 & 0.08 & $0.42^{*}$ & $0.42^{*}$ \\
\hline Forearm circumference maximum $(\mathrm{cm})$ right side & 0.32 & 0.25 & $0.41^{*}$ & $0.39 *$ \\
\hline Forearm circumference minimum $(\mathrm{cm})$ right side & $0.70^{* * *}$ & $0.61^{* *}$ & 0.10 & 0.07 \\
\hline Forearm circumference maximum $(\mathrm{cm})$ left side & 0.19 & 0.15 & $0.54^{* *}$ & $0.53^{* *}$ \\
\hline Forearm circumference minimum $(\mathrm{cm})$ left side & $0.57^{* *}$ & $0.49^{*}$ & 0.33 & 0.33 \\
\hline Hand circumference $(\mathrm{cm})$ dominant side & $0.44^{*}$ & $0.44^{*}$ & $0.44^{*}$ & $0.40^{*}$ \\
\hline Thigh circumference $(\mathrm{cm})$ right side & 0.22 & 0.14 & $0.48^{*}$ & $0.44^{*}$ \\
\hline Thigh circumference $(\mathrm{cm})$ left side & 0.19 & 0.11 & $0.45^{*}$ & $0.42^{*}$ \\
\hline Calf circumference $(\mathrm{cm})$ right side & $0.62^{* *}$ & $0.52^{* *}$ & $0.40^{*}$ & 0.37 \\
\hline Calf circumference $(\mathrm{cm})$ left side & $0.58^{* *}$ & $0.50^{* *}$ & $0.44^{*}$ & $0.42^{*}$ \\
\hline Lower leg circumference $(\mathrm{cm})$ minimum right side & $0.40^{*}$ & $0.42^{*}$ & 0.06 & 0.08 \\
\hline Lower leg circumference $(\mathrm{cm})$ minimum left side & 0.32 & $0.38^{*}$ & 0.20 & 0.24 \\
\hline Anthr. foot length $(\mathrm{cm})$ & 0.15 & 0.11 & -0.17 & -0.15 \\
\hline Techn. foot length $(\mathrm{cm})$ & $0.41^{*}$ & 0.36 & 0.04 & 0.06 \\
\hline Foot width $(\mathrm{cm})$ & $0.44^{*}$ & $0.46^{*}$ & 0.22 & 0.24 \\
\hline Foot circumference $(\mathrm{cm})$ right side & 0.36 & $0.44^{*}$ & 0.05 & 0.08 \\
\hline Foot circumference $(\mathrm{cm})$ left side & 0.33 & $0.41^{*}$ & 0.08 & 0.11 \\
\hline Weight (kg) & 0.46 & 0.38 & 0.36 & 0.34 \\
\hline Body fat (\%)Caliper & 0.10 & -0.08 & 0.21 & 0.21 \\
\hline Body fat (kg) Caliper & 0.19 & 0.01 & 0.22 & 0.23 \\
\hline $\mathrm{BMI}\left(\mathrm{kg} / \mathrm{m}^{2}\right)$ & 0.28 & 0.21 & $0.62^{* *}$ & $0.58^{* *}$ \\
\hline Quetelet-I. (g/cm) & $0.41^{*}$ & 0.32 & $0.52^{* *}$ & $0.49^{*}$ \\
\hline Rohrer-I. $\left(\mathrm{g} / \mathrm{cm}^{3}\right)$ & 0.12 & 0.07 & $0.64^{* *}$ & $0.59^{* *}$ \\
\hline Livi-I. $\left({ }^{3} \sqrt{\mathrm{g}} / \mathrm{cm}\right)$ & 0.12 & 0.07 & $0.64^{* *}$ & $0.59^{* *}$ \\
\hline $\mathrm{HWR}\left(\mathrm{cm} / 3^{3} \sqrt{\mathrm{kg}}\right)$ & -0.11 & -0.06 & $-0.64^{* * *}$ & $-0.59 * *$ \\
\hline Pelidisi-I. $\left({ }^{3} \sqrt{ }(1 / 10 \mathrm{~kg} / \mathrm{cm})\right)$ & 0.09 & 0.10 & $0.51^{* *}$ & $0.45^{*}$ \\
\hline Broca-I. (\%) & 0.25 & 0.18 & $0.63^{* *}$ & $0.59^{* *}$ \\
\hline Triceps skinfold $(\mathrm{mm})$ right side & 0.18 & 0.08 & 0.14 & 0.07 \\
\hline Triceps skinfold $(\mathrm{mm})$ left side & 0.01 & -0.08 & 0.07 & 0.02 \\
\hline Forearm skinfold $(\mathrm{mm})$ non dominant side & 0.32 & $0.39^{*}$ & 0.25 & 0.29 \\
\hline
\end{tabular}




\begin{tabular}{|c|c|c|c|c|}
\hline \multirow{2}{*}{$\begin{array}{l}\text { r } \\
\text { Parameter }\end{array}$} & \multicolumn{2}{|c|}{ Shot putter } & \multicolumn{2}{|c|}{ Javelin thrower } \\
\hline & $\begin{array}{c}\text { best } \\
\text { shot put } \\
\text { distance }\end{array}$ & $\begin{array}{l}\text { current } \\
\text { shot put } \\
\text { distance }\end{array}$ & $\begin{array}{l}\text { best } \\
\text { throw } \\
\text { width }\end{array}$ & $\begin{array}{c}\text { current } \\
\text { throw } \\
\text { width }\end{array}$ \\
\hline Suprailiac skinfold $(\mathrm{mm})$ right side & 0.10 & -0.08 & 0.23 & 0.25 \\
\hline Suprailiac skinfold (mm) left side & -0.01 & -0.18 & 0.22 & 0.25 \\
\hline Subscapular skinfold non dominant side ( $\mathrm{mm})$ & 0.35 & 0.18 & 0.19 & 0.16 \\
\hline Thigh skinfold ( $\mathrm{mm}$ ) non dominant side & -0.32 & $-0.43^{*}$ & 0.12 & 0.09 \\
\hline Calf skinfold $(\mathrm{mm})$ non dominant side & $-0.41^{*}$ & $-0.50^{*}$ & -0.03 & -0.03 \\
\hline Head height $(\mathrm{cm})$ & 0.34 & $0.39 *$ & 0.02 & -0.03 \\
\hline Neck length (cm) & 0.02 & -0.08 & -0.10 & -0.10 \\
\hline Arm length $(\mathrm{cm})$ & 0.33 & 0.32 & -0.03 & 0.03 \\
\hline Length of upper arm and forearm $(\mathrm{cm})$ & 0.36 & 0.31 & 0.04 & 0.06 \\
\hline Upper arm length (cm) & $0.49 *$ & $0.47^{*}$ & 0.30 & 0.37 \\
\hline Forearm length $(\mathrm{cm})$ & 0.08 & 0.02 & -0.20 & -0.24 \\
\hline Hand length $(\mathrm{cm})$ & 0.03 & 0.14 & -0.10 & -0.03 \\
\hline Leg length $(\mathrm{cm})$ & 0.28 & 0.30 & -0.33 & -0.30 \\
\hline Length of thigh and calf $(\mathrm{cm})$ & 0.24 & 0.26 & -0.31 & -0.28 \\
\hline Thigh length $(\mathrm{cm})$ & 0.24 & 0.28 & -0.17 & -0.12 \\
\hline Calf length $(\mathrm{cm})$ & 0.20 & 0.17 & -0.37 & -0.36 \\
\hline Metrik-Index/CONRAD & 0.01 & -0.005 & $0.56^{* *}$ & $0.52^{* *}$ \\
\hline Plastik-Index/CONRAD & $0.55^{* *}$ & $0.52^{* *}$ & $0.41 *$ & 0.36 \\
\hline Pyknomorphy/Knussmann & 0.02 & 0.00 & $0.48^{*}$ & $0.46^{*}$ \\
\hline Makrosomia/Knussmann & $0.58^{* *}$ & $0.58^{* *}$ & 0.15 & 0.10 \\
\hline Endomorphy/PARNELL & 0.09 & -0.05 & 0.17 & 0.15 \\
\hline Mesomorphy/PARNELL & -0.24 & -0.19 & $0.60^{* *}$ & $0.59^{* *}$ \\
\hline Ektomorphy/PARNELL & -0.05 & -0.04 & $-0.60^{* *}$ & $-0.55^{* *}$ \\
\hline Endomorphy/Heath-Carter & 0.12 & -0.06 & 0.21 & 0.18 \\
\hline Mesomorphy/Heath-Carter & 0.04 & 0.00 & $0.55^{* *}$ & $0.54^{* *}$ \\
\hline Ektomorphy/Heath-Carter & -0.09 & -0.04 & $-0.65^{* * *}$ & $-0.60^{* *}$ \\
\hline AKS-Index & 0.14 & 0.13 & $0.66^{* * *}$ & $0.60^{* *}$ \\
\hline
\end{tabular}




\section{DISCUSSION}

In an international comparison of body heights and some other selected anthropometric parameters of the investigated shot putters and javelin throwers they rank in the span of the available literature data, as the following table illustrates.

CARTER established in 1984 a common somatotype for the top athletes who participated in the Olympics in Mexico City in 1968 and the Olympics of Montreal in 1976. For the shot putters a somatotype of $3.2-7.1-1.1$, for the javelin throwers of $2.3-5.9-2.1$ was found. In comparison, the higher class shot putters of the present study achieved a mean somatotype of $2.9-5.6-1.1$ and the higher class javelin throwers of $2.1-4.9-1.8$. This results in the greatest variations in both disciplines for the mesomorphic component.

As shot putters and javelin throwers of the present investigation were rather young, there is still a significant development potential for the mesomorphic component. The Olympians were usually about 28 years old on average.

An important result of the present study is a clear sports anthropological picture of the types of constitution for the junior javelin throwers and junior shot putters: While the shot putter is large and heavy, the javelin thrower appears comparatively smaller, lighter and more athletic with higher values of mesomorphy. 
Table 3. Comparison of selected anthropometric parameters of the investigated shot putters and javelin throwers with available international data

\begin{tabular}{|c|c|c|c|c|c|c|c|c|c|}
\hline \multirow[b]{2}{*}{ Parameter } & \multicolumn{5}{|c|}{ Shot putters } & \multicolumn{4}{|c|}{ Javelin throwers } \\
\hline & Tittel & Tanner & $\begin{array}{c}\text { Correnti/ } \\
\text { Zauli }\end{array}$ & $\begin{array}{l}\text { Tittel et } \\
\text { al. }\end{array}$ & $\begin{array}{l}\text { This } \\
\text { study }\end{array}$ & Tanner & $\begin{array}{c}\text { Correnti/ } \\
\text { Zauli }\end{array}$ & $\begin{array}{l}\text { Tittel et } \\
\text { al. }\end{array}$ & $\begin{array}{l}\text { This } \\
\text { study }\end{array}$ \\
\hline Height (cm) & 185.1 & 190.8 & 187.1 & 194.5 & 189.8 & 186.5 & 182.3 & 183.8 & 183.3 \\
\hline Weight (kg) & 92.2 & 105.0 & 100.3 & 114.3 & 106.7 & 92.9 & 87.3 & 83.9 & 86.6 \\
\hline $\begin{array}{l}\text { Sitting height } \\
(\mathrm{cm})\end{array}$ & & 100.9 & & 97.6 & 97.8 & 98.1 & & 94.2 & 95.6 \\
\hline $\begin{array}{l}\text { Upper arm length } \\
(\mathrm{cm})\end{array}$ & & & 36.3 & 35.1 & 36.7 & & 35.1 & 36.3 & 35.5 \\
\hline $\begin{array}{l}\text { Forearm length } \\
(\mathrm{cm})\end{array}$ & & & & 27.8 & 27.6 & & & 26.1 & 26.7 \\
\hline Hand length $(\mathrm{cm})$ & & & & 21.5 & 20.6 & & & 20.5 & 20.0 \\
\hline Arm length $(\mathrm{cm})$ & 85.9 & 84.2 & & 74.4 & 84.9 & 83.6 & & 82.9 & 82.2 \\
\hline Thigh length $(\mathrm{cm})$ & & & & 53.8 & 55.3 & & & 50.1 & 52.7 \\
\hline Calf length $(\mathrm{cm})$ & & & & 45.3 & 43.8 & & & 40.8 & 42.4 \\
\hline Leg length $(\mathrm{cm})$ & 88.7 & 89.8 & & 99.1 & 99.1 & 88.4 & & 90.9 & 95.0 \\
\hline Foot length $(\mathrm{cm})$ & & & & 28.9 & 26.9 & & & 27.7 & 26.8 \\
\hline $\begin{array}{l}\text { Shoulder width } \\
(\mathrm{cm})\end{array}$ & 42.5 & 46.0 & 44.0 & 40.5 & 46.3 & 45.3 & 40.8 & 41.6 & 45.2 \\
\hline Chest width $(\mathrm{cm})$ & & 31.8 & 31.3 & 34.0 & 33.0 & 30.9 & 29.4 & 31.4 & 30.0 \\
\hline Chest depth $(\mathrm{cm})$ & & 22.5 & 22.5 & 26.0 & 22.3 & 22.8 & & 21.9 & 20.5 \\
\hline $\begin{array}{l}\text { Chest circumfer- } \\
\text { ence, respiratory } \\
\text { centre }(\mathrm{cm})\end{array}$ & & & & 115.0 & 112.7 & & & 105.1 & 103.8 \\
\hline $\begin{array}{l}\text { Chest circumfer- } \\
\text { ence, in inspira- } \\
\text { tion }(\mathrm{cm})\end{array}$ & & & & 118.0 & 115.0 & & & 109.1 & 106.5 \\
\hline $\begin{array}{l}\text { Chest circumfer- } \\
\text { ence, in exspira- } \\
\text { tion }(\mathrm{cm})\end{array}$ & & & & 113.0 & 111.8 & & & 102.3 & 102.1 \\
\hline $\begin{array}{l}\text { Upper arm cir- } \\
\text { cumference }(\mathrm{cm})\end{array}$ & & 37.6 & 40.7 & 37.0 & 38.1 & 35.0 & & 32.6 & 35.6 \\
\hline $\begin{array}{l}\text { Thigh circum- } \\
\text { ference }(\mathrm{cm})\end{array}$ & & 67.0 & & 65.0 & 66.3 & 62.1 & & 57.5 & 61.1 \\
\hline $\begin{array}{l}\text { Calf circum- } \\
\text { ference }(\mathrm{cm})\end{array}$ & & & 41.3 & 45.0 & 43.0 & 41.4 & & 39.2 & 39.5 \\
\hline $\begin{array}{l}\text { Epiphysis width } \\
\text { Humerus (cm) }\end{array}$ & & 8.1 & 7.7 & & 7.1 & 7.5 & & & 6.7 \\
\hline $\begin{array}{l}\text { Epiphysis width } \\
\text { Femur }(\mathrm{cm})\end{array}$ & & 11.1 & & & 10.6 & 10.3 & & & 9.9 \\
\hline BMI $\left(\mathrm{kg} / \mathrm{m}^{2}\right)$ & 26.9 & 29.1 & 28.7 & 30.4 & 28.8 & 26.9 & 26.4 & 25.0 & 24.7 \\
\hline
\end{tabular}




\section{REFERENCES}

1. Carter J.E.L. (1984). Physical Structure of Olympic Athletes. Part II. Kinanthropometry of Olympic Athletes. Medicine and Sport Science 18. Basel, München, Paris, London, New York, Tokio, Sydney, Verlag S. Karger.

2. Conrad K. (1963). Der Konstitutionstypus. Berlin, Springer Verlag.

3. Correnti V., Zauli B. (1964). Olympionici 1960. Rom, Marves.

4. Heath B.H., Carter L.J.E. (1967). A modified somatotype method. Am J Phys Anthrop, 27, 57-74.

5. Heath B.H., Carter L.J.E. (1990). Somatotyping- development and applications. Cambridge Studies in Biological Anthropology. Great Britain, Redwood Press.

6. Hirata, K.-I. (1979). Selection of Olympic champions. Toyota (Japan), Chukyo University Press.

7. Knußmann R. (1996). Vergleichende Biologie des Menschen. Lehrbuch der Anthropologie. Stuttgart, Gustav Fischer Verlag.

8. Kretschmer E. (1921). Körperbau und Charakter. Berlin, Springer Verlag.

9. Martin R., Knussmann R. (1988). Anthropologie. Handbuch. Band I. Stuttgart, Fischer Verlag.

10. Parnell R.W. (1954). Somatotyping by physical anthropometry. Am J Phys Anthrop, 12, 209-239.

11. Raschka C. (2006). Sportanthropologie. Köln, Sportverlag Strauß.

12. Tanner J.M. (1964). The physique of the Olympic athlete. A study of 137 track and field athletes at the Olympic Games, Rome 1960 and a comparison with weight lifters and wrestlers. London, Allen \& Unwin.

13. Tittel K., Wutscherk H. (1972). Sportanthropometrie. Leipzig, Barth.

\section{Adress for correspondence}

Priv.-Doz. Dr.med. Dr.rer.nat. Dr.Sportwiss. Christoph Raschka

Institute of Sports Sciences, Julius-Maximilians-University, Germany

Judenbühlweg 11

D-97082 Würzburg

E-mail: christoph.raschka@uni-wuerzburg.de 